\title{
Effect of Pioglitazone on Perihematomal Edema in Intra- cerebral Hemorrhage Mouse Model by Regulating NLRP3 Expression and Energy Metabolism
}

\author{
Hoon Kim, Jung Eun Lee, ${ }^{2}$ Hyun Ju Yoo, ${ }^{3}$ Jae Hoon Sung, ${ }^{2}$ Seung Ho Yang ${ }^{4}$ \\ Department of Neurosurgery, Bucheon St. Mary's Hospital, College of Medicine, The Catholic University of Korea, Seoul, Korea \\ Department of Neurosurgery, ${ }^{2}$ St. Vincent's Hospital, College of Medicine, The Catholic University of Korea, Seoul, Korea \\ Biomedical Research Center, ${ }^{3}$ Asan Medical Center, University of Ulsan College of Medicine, Seoul, Korea \\ Department of Neurosurgery, ${ }_{4}^{4}$ Cell Death Disease Research Center, St. Vincent's Hospital, College of Medicine, The Catholic University of \\ Korea, Seoul, Korea
}

Objective : Cerebral edema is the predominant mechanism of secondary inflammation after intracerebral hemorrhage (ICH). Pioglitazone, peroxisome proliferator-activated receptor gamma agonist has been shown to play a role in regulation of central nervous system inflammation. Here, we examined the pharmacological effects of pioglitazone in an ICH mouse model and investigated its regulation on NLRP3 inflammasome and glucose metabolism.

Methods : The ICH model was established in C57 BL/6 mice by the stereotactical inoculation of blood ( $30 \mu \mathrm{L})$ into the right frontal lobe. The treatment group was administered i.p. pioglitazone $(20 \mathrm{mg} / \mathrm{kg})$ for 1, 3, and 6 days. The control group was administered i.p. phosphate-buffered saline for 1, 3, and 6 days. We investigated brain water contents, NLRP3 expression, and changes in the metabolites in the ICH model using liquid chromatography-tandem mass spectrometry.

Results : On day 3, brain edema in the mice treated with pioglitazone was decreased more than that in the control group. Expression levels of NLRP3 in the ICH model treated with pioglitazone were decreased more than those of the control mice on days 3 and 7. The pioglitazone group showed higher levels of glycolytic metabolites than those in the ICH mice. Lactate production was increased in the $\mathrm{ICH}$ mice treated with pioglitazone.

Conclusion : Our results demonstrated less brain swelling following $\mathrm{ICH}$ in mice treated with pioglitazone. Pioglitazone decreased NLRP3-related brain edema and increased anaerobic glycolysis, resulting in the production of lactate in the ICH mice model. NLRP3 might be a therapeutic target for $\mathrm{ICH}$ recovery.

Key Words : Cerebral hemorrhage · Brain edema · Inflammasomes · Pioglitazone · Lactates.

- Received : February 27, 2020 • Revised : April 5, 2020 • Accepted : May 26, 2020

- Address for reprints : Seung Ho Yang

Department of Neurosurgery, Cell Death Disease Research Center, St. Vincent's Hospital, College of Medicine, The Catholic University of Korea, 93 Jungbu-daero, Paldalgu, Suwon 16247, Korea

Tel : +82-31-249-8303, Fax : +82-31-245-5208, E-mail : 72ysh@catholic.ac.kr, ORCID : https://orcid.org/0000-0002-3490-1064

This is an Open Access article distributed under the terms of the Creative Commons Attribution Non-Commercial License (http://creativecommons.org/licenses/by-nc/4.0) which permits unrestricted non-commercial use, distribution, and reproduction in any medium, provided the original work is properly cited. 


\section{INTRODUCTION}

Intracerebral hemorrhage (ICH), accounts for $10-15 \%$ of all stroke types with high morbidity and mortality ${ }^{24)}$. Hematoma formation and cerebral edema begin within hours by extravasation of blood products into the brain parenchyma and lasts for weeks ${ }^{17)}$. Thus, increasing intracranial pressure leads to neurological deterioration $^{23)}$.

Neuroinflammation is known as a major contributor and hallmark of brain injury caused by $\mathrm{ICH}^{3,38)}$. NLRP3 is activated in response to pathogens, several pathogen-associated molecular patterns, danger-associated molecular patterns, and environmental irritants ${ }^{31}$. Activation of NLRP3 is a critical component of the inflammasome and plays an independent role in injury signaling, apart from other inflammasome components $^{32,34)}$. Finally, it can lead to cell death ${ }^{20,39)}$. However, inhibition of the NLRP3 inflammasome has been shown to suppress the inflammatory response and reduce cell death ${ }^{21,43)}$. Recently, pioglitazone, peroxisome proliferator-activated receptor gamma (PPAR- $\gamma$ ) agonist, reduced cerebral edema and immune response after traumatic brain injury by downregulating the effects of NLRP ${ }^{15)}$.

Metabolites have an important role in biology as structures of the genome, proteomes, and cell membranes. Additionally, they have other functions as signaling molecules, energy sources, and metabolic intermediates. Mass spectroscopy (MS) remains the most favored technology for metabolomics due to its wide dynamic range and good sensitivity in the nanomolar range ${ }^{19)}$. However, few studies have reported metabolomic analyses in hemorrhagic stroke patients ${ }^{4}$. In the present study, we investigated the effect of pioglitazone on NLRP3-related brain edema and glucose metabolism in an animal model of ICH.

\section{MATERIALS AND METHODS}

This study was approved by Institutional Review Board of The Catholic University of Korea St. Vincent's Hospital Institutional Animal Care and Use Committee (IRB No. 17-8).

\section{ICH mouse model}

Six-week-old male C57/BL mice (Central Laboratory Animals, Seoul, Korea) were used. The mice were housed in a standardized animal room (lights on 7 am to $7 \mathrm{pm}$, room temperature $22 \pm 2^{\circ} \mathrm{C}$ ). The mice were anesthetized with isoflurane and a midline incision was made in the head. Then, they were inoculated stereotactically with arterial blood (30 $\mu \mathrm{L})$ into the right frontal lobe $(2 \mathrm{~mm}$ lateral and $1 \mathrm{~mm}$ posterior to the bregma at a depth of $2.5 \mathrm{~mm}$ from the skull) using a sterile Hamilton syringe fitted with a 26-gauge needle (Hamilton, Reno, NV, USA) and a microinfusion pump (Harvard Apparatus, Holliston, MA, USA). The needle was left in place for an additional 5 minutes after injection to prevent possible leakage. It was slowly withdrawn within 2 minutes ${ }^{28)}$. Following the surgery, the skull hole was sealed with bone wax and the incision was closed with sutures and the mice were allowed to recover. To avoid postsurgical dehydration, $0.5 \mathrm{~mL}$ of normal saline was given to each mouse by subcutaneous injection immediately after surgery.

\section{Sample preparation for the assessment of brain edema}

After the mice were euthanized by $\mathrm{CO}_{2}$ inhalation, the brain was removed and the cerebrum was dissected from the brain stem. The wet weight of the cerebrum was measured and the cerebrum was dried in a dry oven at $100^{\circ} \mathrm{C}$ for 30 hours. The dry weight was then determined. The water content of the brain was calculated as follows : water content $=$ [(wet weight - dry weight $) /$ wet weight $] \times 100 \%{ }^{9)}$.

\section{Western blot analysis}

Total protein was extracted using a PhosSTOP EASYpack (Roche, Mannheim, Germany) according to the manufacturer's instructions. The proteins were separated by SDS-PAGE, transferred to nitrocellulose membranes, and detected with antibodies against NLRP3 (Cell Signaling Technology, Danvers, MA, USA) and $\beta$-actin (Sigma-Aldrich, Co., St. Louis, MO, USA). Immunoreactivity was detected using the ECL chemiluminescence system and quantified using an imaging densitometer. The density of each band was quantified using Quantity One software (Bio-Rad, Hercules, CA, USA).

\section{Liquid chromatography-tandem mass spectrom- etry (LC-MS/MS)}

Brain tissue (50-100 mg) was homogenized using a TissueLyzer (Qiagen, Germantown, MD, USA) with $400 \mu \mathrm{L}$ of chloroform/methanol (2/1). The homogenate was incubated for 20 
minutes at $4^{\circ} \mathrm{C}$. Glutamine- $\mathrm{d}_{4}$ was added to each sample as an internal standard after incubation and mixed well. The sample was then centrifuged at $13000 \mathrm{rpm}$ for 10 minutes. The supernatant was collected and $100 \mu \mathrm{L}$ of $\mathrm{H}_{2} \mathrm{O}$ was added. The sample was mixed vigorously and centrifuged at $4000 \mathrm{rpm}$ for 20 minutes. The upper phase was taken and dried under vacuum. The dried sample was stored at $-20^{\circ} \mathrm{C}$ and reconstituted with $40 \mu \mathrm{L}$ of $\mathrm{H}_{2} \mathrm{O} /$ acetonitrile (50/50 v/v) prior to LC-MS/MS analysis

Metabolites related to energy metabolism were analyzed with an LC-MS/MS equipped with a 1290 HPLC (Agilent, Santa Clara, CA, USA), Qtrap 5500 (ABSciex, Flamingham, MA, USA) and a 50×2 mm reverse phase column (Synergi Fusion-RP). Then, $3 \mu \mathrm{L}$ was injected into the LC-MS/MS system and ionized with a turbo spray ionization source. Ammonium acetate $\left(5 \mathrm{mM}\right.$ in $\mathrm{H}_{2} \mathrm{O}$ ) and $5 \mathrm{mM}$ ammonium acetate in acetonitrile were used as mobile phases $\mathrm{A}$ and $\mathrm{B}$, respectively. The separation gradient was as follows : hold at $0 \%$ B for 5 minutes, $0 \%$ to $90 \%$ B for 2 minutes, hold at $90 \%$ for 8 minutes, $90 \%$ to $0 \% \mathrm{~B}$ for 1 minute, then hold at $0 \% \mathrm{~B}$ for 9 minutes. The LC flow rate was set at $70 \mu \mathrm{L} / \mathrm{min}$, except for $140 \mu \mathrm{L} / \mathrm{min}$ from 7 to 15 minutes. The column temperature was maintained at $23^{\circ} \mathrm{C}$. Multiple reaction monitoring was used in the negative ion mode. Extracted ion chromatogram (EIC) corresponding to the specific transition for each metabolite was used for quantitation. The area under the curve of each EIC was normalized to the EIC of the internal standard and the ratio was used for relative comparisons.

\section{RESULTS}

\section{ICH mouse model}

We established an ICH mouse model through intracranial injection of autologous whole blood (30 $\mu \mathrm{L})$ (Fig. 1). A total of 30 mice were randomly assigned into the control and treatment groups. Mice in the treatment group were administered pioglitazone i.p. $(20 \mathrm{mg} / \mathrm{kg})$ for 1,3 , and 6 days. Mice in the control group were administered phosphate-buffered saline i.p. for 1, 3, and 6 days. The mice were sacrificed on days 1, 3, and 7 for analysis (Fig. 2).

\section{Pioglitazone administration reduces brain edema}

We compared the water contents of the ipsilateral and contralateral hemispheres in the ICH mice and ICH mice treated with pioglitazone. On day 3 , brain edema in the ipsilateral hemispheres of mice treated with pioglitazone (77.69 $\pm 0.50 \%)$ was decreased more than that of the ipsilateral hemispheres in the ICH mice $(79.30 \pm 0.55 \%)$ ( $p=0.0001)$. The same finding was made in the contralateral hemispheres. On day 7 , the brain edema in mice treated with pioglitazone was decreased more than that in the ICH mice. Brain edema in the ipsilateral

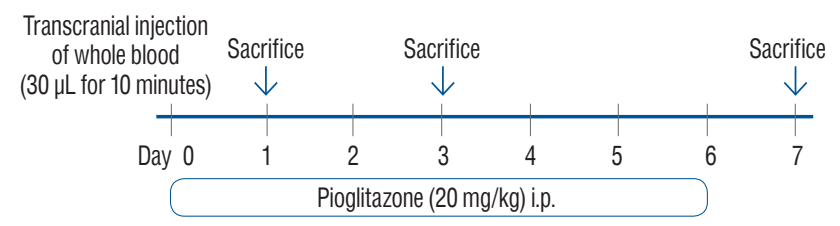

Fig. 2. Treatment schedule.

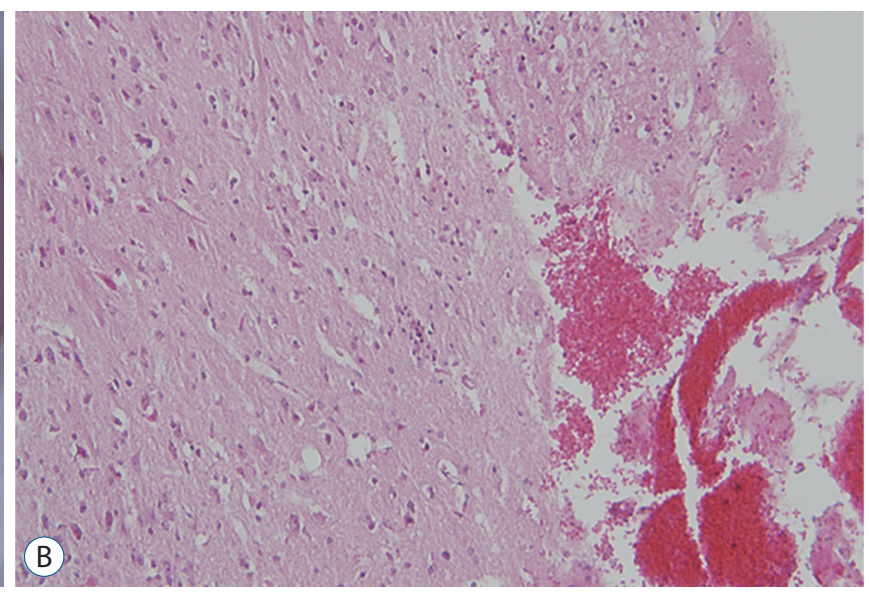

(A)

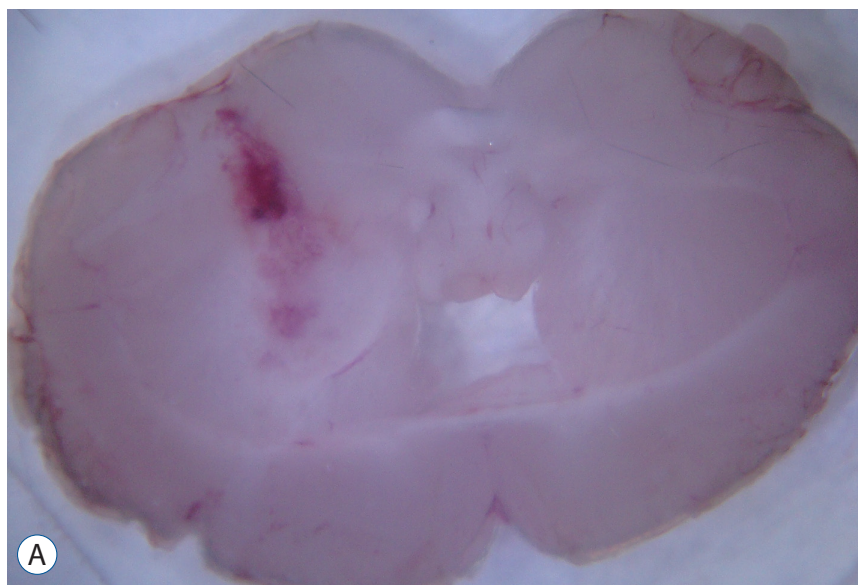

(B)

Fig. 1. Establishment of intracranial hemorrhage (ICH) models (hematoxylin and eosin, $\mathrm{H} \& \mathrm{E})$. Coronal section of the whole brain (A, scale bar=3 mm) and magnification of the hematoma $(B, \times 200)$. 
hemispheres $(75.11 \pm 0.35 \%)$ on day 7 was decreased more than that of the ipsilateral hemispheres $(77.69 \pm 0.50 \%)$ on day 3 in the pioglitazone-treated group $(p<0.0001)$ (Fig. 3).

\section{Pioglitazone administration reduces NLRP3}

We compared the expression levels of NLRP3 in the ipsilateral hemispheres among the groups. The expression levels of NLRP3 in the ICH mice treated with pioglitazone were decreased more than those of the ICH mice on day $7(p=0.025)$ (Fig. 4).

\section{Pioglitazone administration modulates metabo- lism}

Brain tissues were obtained from the control mice, ICH mice, and ICH mice treated with pioglitazone on day 7 and analyzed by LC-MS/MS. The values are calculated with an equation (peak area of analyte/peak area of internal standard).

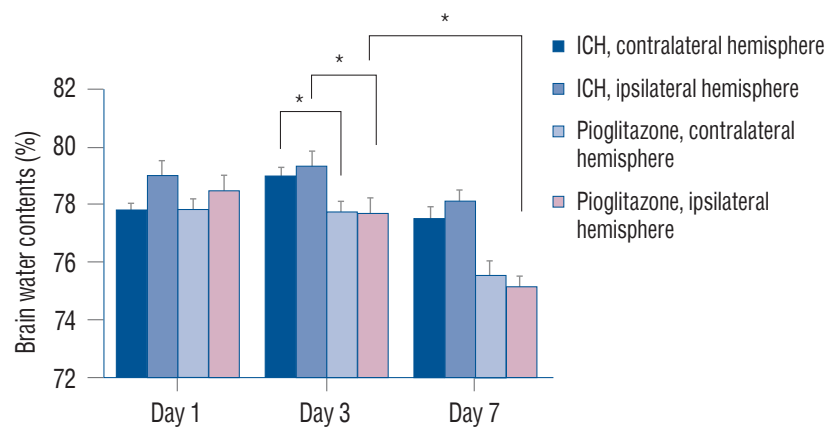

Fig. 3. Comparison of brain water contents. The water contents of both hemispheres in ICH mice treated with pioglitazone were lower than those in $\mathrm{ICH}$ mice on days 3 and 7. In the treatment group, the water content of the ipsilateral hemispheres on day 7 was decreased more than on day 3 ( $n=7$, each group). ${ }^{*} p<0.05$. ICH : intracranial hemorrhage.
Increased glycolysis was observed in the brains of the ICH mice treated with pioglitazone for 6 days. Increased glucose availability was accompanied by an increase in glycolytic intermediates (glucose-6-phosphate, 19.76 4 4.17; fructose-1,6biphosphate, 10.96 \pm 1.68 ; and pyruvate, 2.42 \pm 0.16$)$ and increased lactate production (42.53 \pm 2.88 ) (Fig. 5A). Differences in the tricarboxylic acid (TCA) cycle intermediates (citrate, $\alpha$-ketoglutarate, succinate, fumarate, and malate) were not significant between the ICH mice and the ICH mice treated with pioglitazone. Various metabolites, such as adenosine monophosphate, adenosine diphosphate, adenosine triphosphate (ATP), nicotinamide adenine dinucleotide (NADH), reduced $\mathrm{NADH}$, and phosphoenolpyruvate, were investigated. The levels of NADH were lower in ICH mice treated with pioglitazone $(0.07 \pm 0.02)$ than in the ICH mice $(0.21 \pm 0.00)$. No differences in the other metabolites were noted (Fig. 5B).

We also investigated the pentose phosphate pathway (PPP), including ribulose-5-phosphate/ribose-5-phosphate, ribose-1,5-bisphosphate, sedoheptulose-7-phosphate, 6-phosphogluconate, nicotinamide adenine dinucleotide phosphate (NADPH), and reduced NADPH. There were no significant differences in the PPP metabolites between the ICH mice and ICH mice treated with pioglitazone (Fig. 5C).

\section{DISCUSSION}

We investigated pharmacological effects of pioglitazone on NLRP3 expression and perihematomal edema in an ICH mouse model. Pioglitazone is an agonist of the PPAR that regulates lipid metabolism and reduces insulin resistance ${ }^{40)}$.

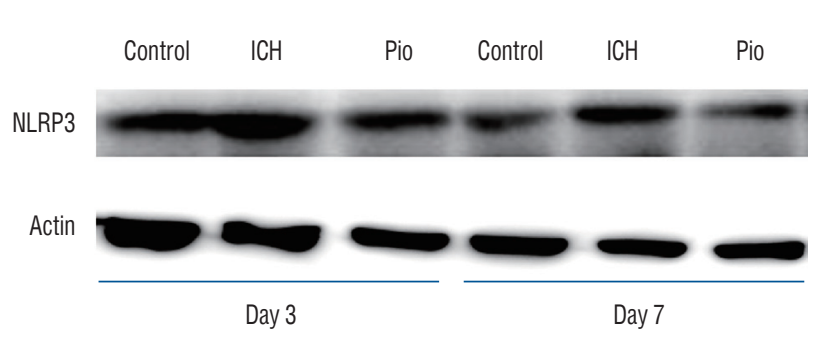

(A)

Fig. 4. Western blot of NLRP3 in the control mice, ICH mice, and ICH mice treated with treated with pioglitazone was decreased more than those in the ICH mice on day 7 (B). ${ }^{*} p<0.05$. ICH : intracranial hemorrhage.

B)

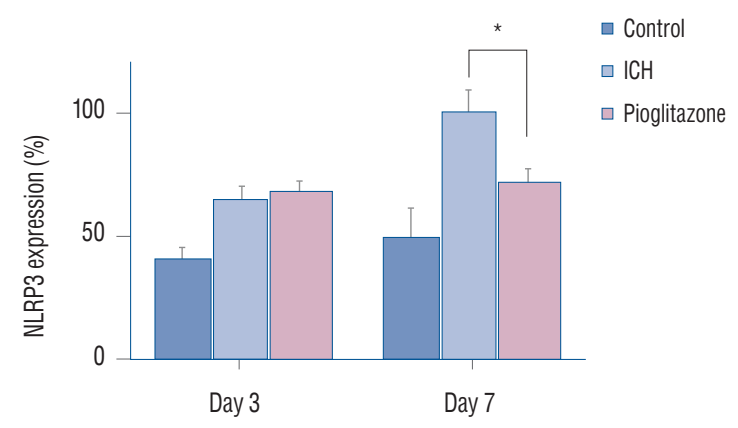


PPAR is a member of the nuclear receptor superfamily. It is expressed in monocytes, macrophages, and microglia. Recent studies have revealed that pioglitazone can reduce macrophage infiltration and the activation of tumor growth factor beta-1, leading to a deterioration in NLRP3 levels and downstream secretion of inflammatory cytokines ${ }^{12,26)}$. Our results demonstrated that the brain swelling following $\mathrm{ICH}$ was less in mice treated with pioglitazone compared to ICH mice. The water content of the ICH hemispheres became greater as time passed. Pioglitazone administration reduced the water content in the ICH mice. The initial bleeding caused mechanical destruction of the brain's cellular structure. Hematoma formation can compress the surrounding brain and increase intracranial pressure, thereby potentially affecting blood flow ${ }^{18)}$. The therapeutic targets of ICH have focused on secondary brain injury because it is reversible. Secondary damage after $\mathrm{ICH}$ is caused by a cascade effect initiated by the primary injury (e.g., mechanical disruption and mass effect), the release of clotting components (e.g., hemoglobin and iron), and the biophysiological response to the hematoma (e.g., inflammation). A pronounced inflammatory response occurs with the activation of resident microglia, the influx of leukocytes into the brain, and the generation of inflammatory mediators $^{22,27,33)}$. The NLRP3 inflammasome can mediate perihematomal neuronal death. It occurs as early as three to six hours after a stroke, especially in $\mathrm{ICH}^{10,44)}$. Inflammation and immunity have protentional roles in cerebral edema and NLRP3 is a well-known component of this cascade ${ }^{37)}$. The NLRP3 inflammasome is associated with ICH-induced secondary injury. Inhibition of NLRP3 may affect the recovery of brain function after $\mathrm{ICH}^{41)}$. We found that the NLRP3 was decreased in pioglitazone-treated ICH mice, suggesting that NLRP3 downregulation could reduce cerebral edema caused by ICH.

We observed that glucose uptake was converted into lactate through hyperglycolysis in the ICH model mice treated with pioglitazone. The levels of glycolytic metabolites were higher
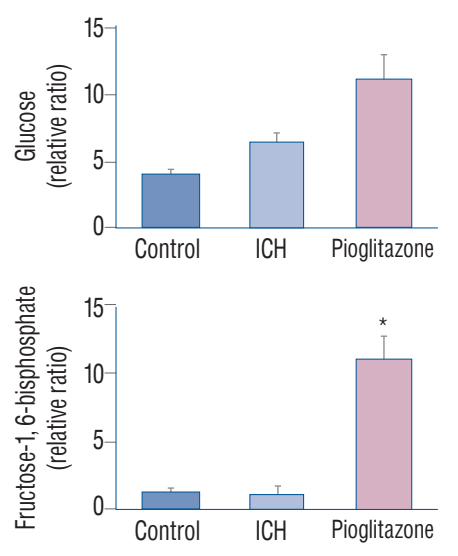

Glucose-6

ate

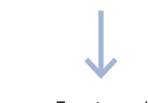

Fructose-1, 6-biphosphate

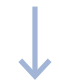

3-phosphoglycerate

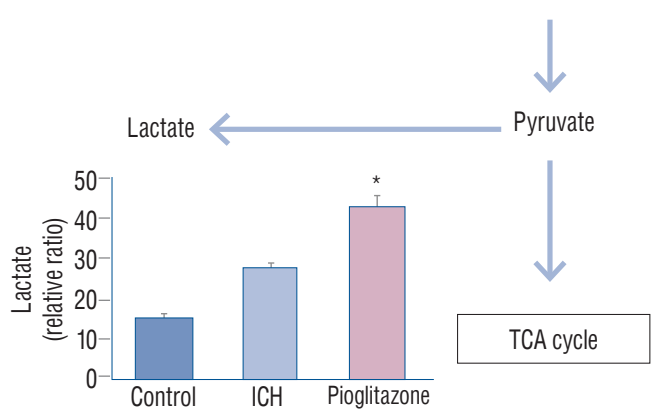

Pyruvate

Lactate

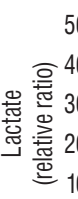

(A)
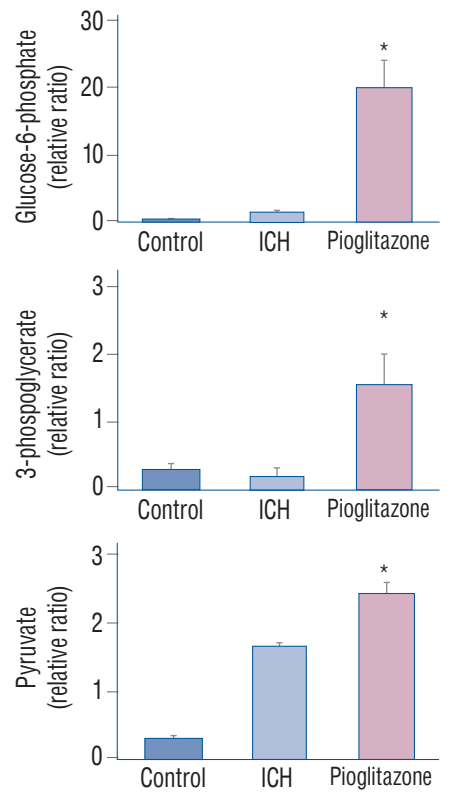

Fig. 5. Liquid chromatography-tandem mass spectrometry analysis. In the glycolysis pathway, the production of glucose-6-phosphate, fructose-1,6biphosphate, pyruvate, and lactate were increased in ICH mice brains treated with pioglitazone compared to those ICH mice (A). In the TCA cycle, the production of nicotinamide adenine dinucleotide was decreased in ICH mice treated with pioglitazone more than that of ICH mice. Differences in other metabolites were not noted (B). In the PPP, there were no significant differences in PPP metabolites between the two groups (C). ${ }^{*} p<0.05$. ICH : intracranial hemorrhage, TCA : tricarboxylic acid, PPP : pentose phosphate pathway. 

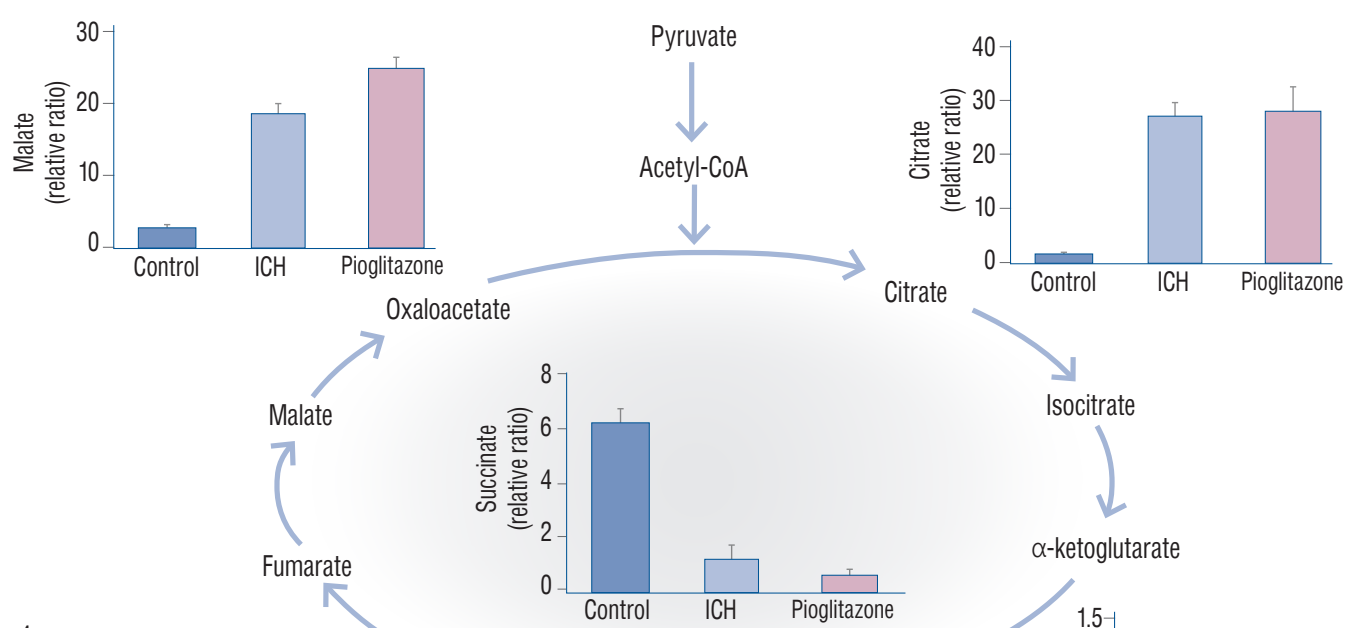

Isocitrate
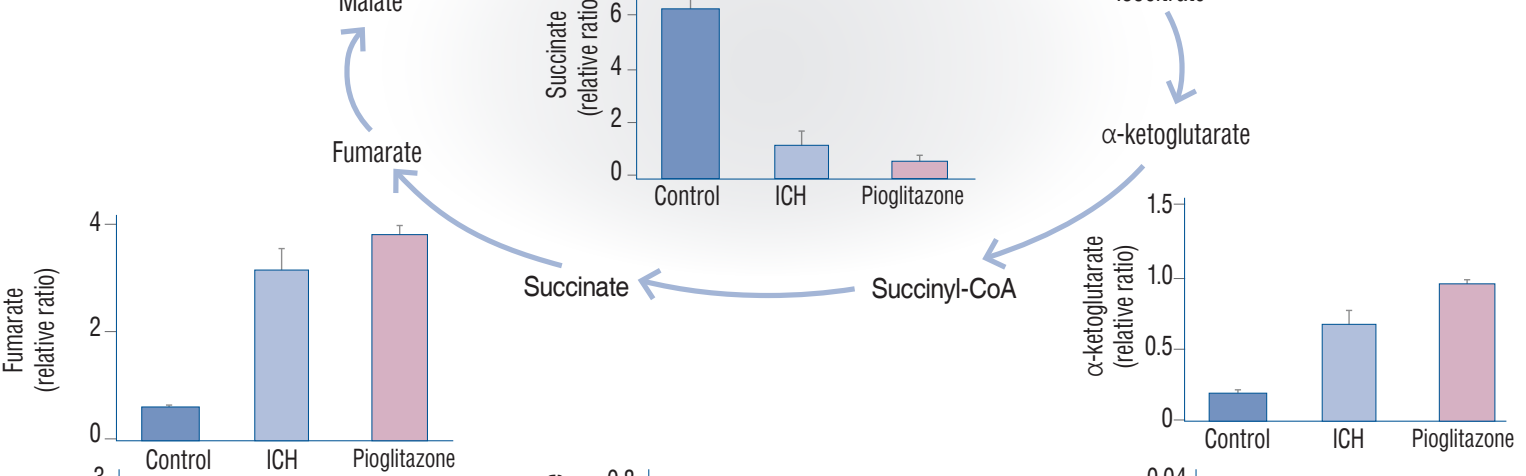

(B)
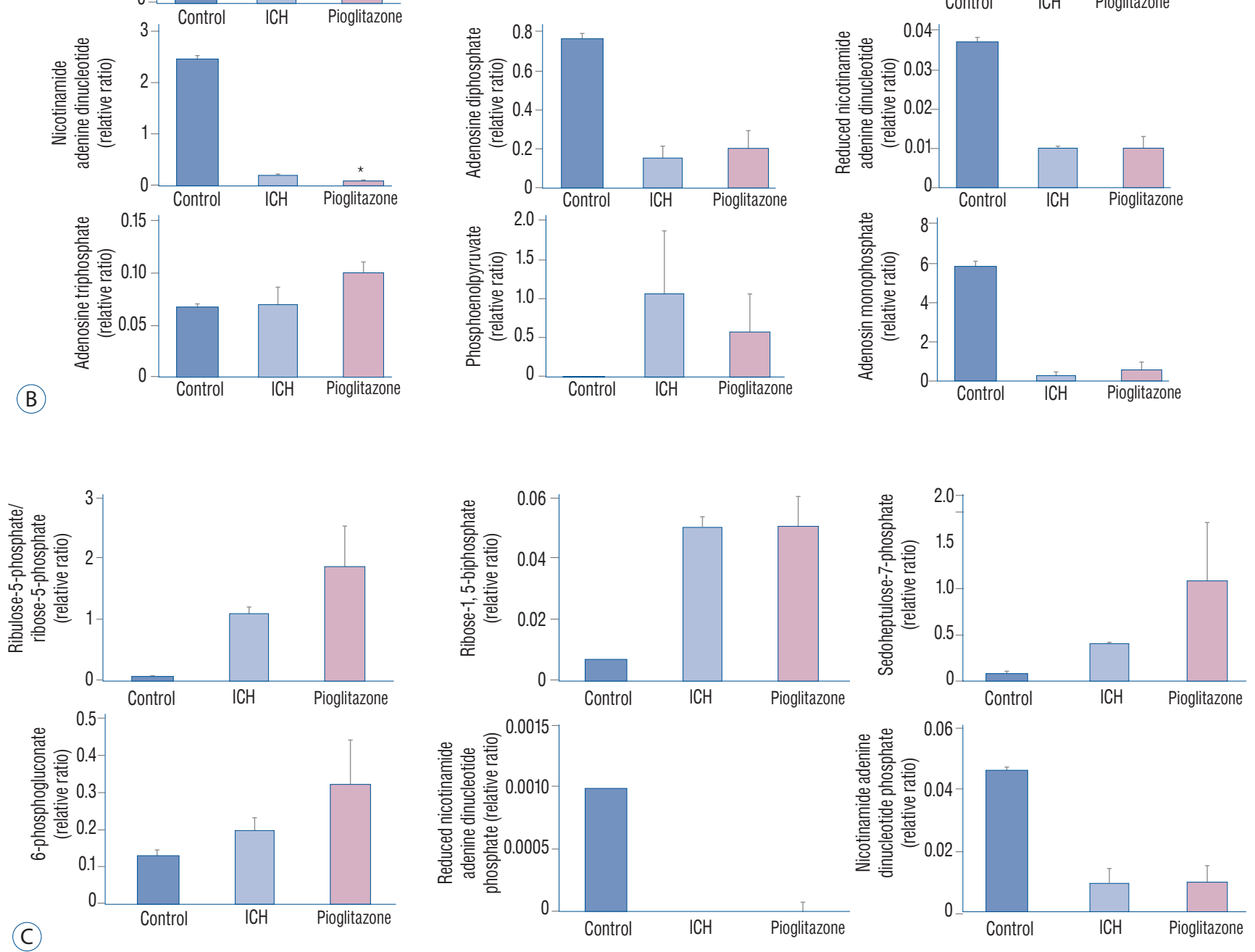

Fig. 5. Continued. 
in ICH mice treated with pioglitazone than in the untreated ICH mice. Cerebral hyperglycolysis is thought to operate to cope with the extreme metabolic demand of the brain cells to restore homeostasis and integrity during recovery from $\mathrm{ICH}$. Pioglitazone administration could compensate oxidative phosphorylation normally and meet an increased energy demand by an increased glycolysis.

Lactate is an important cerebral substrate of glucose. Neuroprotective effects and improvement in cognition following lactate administration have been reported in a traumatic brain injury model ${ }^{25}$. Other reports have revealed reductions in lesion sizes in both stroke and traumatic brain injury (TBI) animal models by lactate administration ${ }^{2,6)}$. Lactate provides an energy source and is involved in cerebral metabolism in the glucose-deprived brain ${ }^{11}$. During neuronal glycolysis, lactate derived from astrocytes is produced at a faster rate, providing a quick and readily available source to meet increasing energy requirements ${ }^{7,30)}$. Lactate-to-pyruvate ratio (LPR) has been found to be an independent predictor of mortality and unfavorable outcome in the largest cohort of TBI patients monitored with microdialysis ${ }^{35}$. Lactate concentration is a highly sensitive indicator of upregulated glycolytic flux but pyruvate levels are necessary in order to differentiate whether the upregulation of the glycolytic flux is anaerobic or an indicator of an increased use of the glycolysis under aerobic conditions. An increased levels of lactate and a high LPR have been highly sensitive predictors of poor outcomes ${ }^{29)}$. Microdialysis can allow for the direct assessment of brain energetic metabolism after ICH. In one study, the effects of pioglitazone on glucose metabolism were investigated in cultured rat neurons and astroglia. Pioglitazone improved aerobic glycolysis and lactate release in the astroglia. These results revealed that pioglitazone may increase the efficiency of glucose metabolism in the damaged brain ${ }^{16)}$. To the best of our knowledge, this is the first report showing that pioglitazone administration can increase levels of pyruvate and lactate in mice brains with ICH. Our results suggest that the production of lactate could supply the energy needs in ICH settings.

TCA cycle not only provides reducing equivalents of the respiratory complexes, it also generates high-energy phosphates. In the presence of oxygen, $\mathrm{NADH}$ are oxidized, leading to the development of an electrochemical gradient across the inner mitochondrial membrane. This electrochemical gradient is utilized by ATP synthase to make ATP. During anoxia, NADH cannot be oxidized by the respiratory complexes; therefore, oxidative phosphorylation ceases ${ }^{8}$. PPP, which produces ribose5-phosphate and NADPH for DNA/RNA and fatty acid synthesis, is an alternative anabolic pathway to the preparatory phase of glycolysis. PPP are highly activated under normoxic conditions, whereas acute hypoxia causes downregulation of PPP metabolites concomitant with upregulation of glycolysis ${ }^{36}$. ATP is increased in the ICH mice treated with pioglitazone, compared with the ICH mice. However, the difference was not statistically significant between them. Although neither the intermediates of the TCA cycle nor the PPP metabolites were affected by pioglitazone administration, the level of NAD decreased. Cellular NAD was shown to be significantly depleted during reperfusion injury after ischemia ${ }^{5,14)}$. Also, exogenous NAD supplementation can increase intracellular NAD levels and reduce reperfusion-induced cell death in primary neuron cultures $^{1,42)}$. NAD is effective only when it is given within two hours after reperfusion ${ }^{13)}$. We removed the ICH brains seven days after the injection of autologous blood for metabolomics analysis. Further study is needed to evaluate the changes in metabolites from the hyperacute stage to the late stage and define the therapeutic window of pioglitazone.

\section{CONCLUSION}

In summary, pioglitazone decreased NLRP3-related brain edema and increased anaerobic glycolysis, resulting in the production of lactate in an ICH mouse model. NLRP3 might be a therapeutic target for ICH recovery. The current study suggests that administration of pioglitazone could be an effective strategy for hemorrhagic stroke.

\section{CONFLICTS OF INTEREST}

No potential conflict of interest relevant to this article was reported.

\section{INFORMED CONSENT}

This type of study does not require informed consent. 


\section{AUTHOR CONTRIBUTIONS}

\author{
Conceptualization : JHS, SHY \\ Data curation : HK, JEL, HJY \\ Formal analysis : HK, SHY \\ Funding acquisition : SHY \\ Methodology : JEL, HJY \\ Project administration : JEL \\ Writing - original draft : HK, JEL \\ Writing - review \& editing: HJY, JHS, SHY
}

\section{ORCID}

$\begin{array}{ll}\text { Hoon Kim } & \text { https://orcid.org/0000-0003-1841-0991 } \\ \text { Jung Eun Lee } & \text { https://orcid.org/0000-0002-8401-2839 } \\ \text { Hyun Ju Yoo } & \text { https://orcid.org/0000-0003-2617-444X } \\ \text { Jae Hoon Sung } & \text { https://orcid.org/0000-0003-3738-6413 } \\ \text { Seung Ho Yang } & \text { https://orcid.org/0000-0002-3490-1064 }\end{array}$

\section{- Acknowledgements}

This study was supported by the National Research Foundation of Korea (NRF)-2016R1D1A1A02937141. The author would like to acknowledge financial support from St. Vincent' s Hospital Research Institute of Medical Science (SVHR-2017$03)$.

\section{References}

1. Alano CC, Garnier P, Ying W, Higashi Y, Kauppinen $T M$, Swanson RA : $N A D+$ depletion is necessary and sufficient for poly(ADP-ribose) polymerase-1-mediated neuronal death. J Neurosci 30 : 2967-2978, 2010

2. Alessandri B, Schwandt E, Kamada $Y$, Nagata M, Heimann A, Kempski 0 : The neuroprotective effect of lactate is not due to improved glutamate uptake after controlled cortical impact in rats. J Neurotrauma 29 : 2181-2191, 2012

3. Aronowski J, Zhao X : Molecular pathophysiology of cerebral hemorrhage: secondary brain injury. Stroke 42 : 1781-1786, 2011

4. Au A : Metabolomics and lipidomics of ischemic stroke. Adv Clin Chem 85 : 31-69, 2018

5. Becatti M, Taddei N, Cecchi C, Nassi N, Nassi PA, Fiorillo C : SIRT1 modulates MAPK pathways in ischemic-reperfused cardiomyocytes. Cell Mol Life Sci 69 : 2245-2260, 2012

6. Berthet C, Castillo X, Magistretti PJ, Hirt L : New evidence of neuro- protection by lactate after transient focal cerebral ischaemia: extended benefit after intracerebroventricular injection and efficacy of intravenous administration. Cerebrovasc Dis 34 : 329-335, 2012

7. Castro MA, Beltrán FA, Brauchi $S$, Concha II : A metabolic switch in brain: glucose and lactate metabolism modulation by ascorbic acid. J Neurochem 110 : 423-440, 2009

8. Chinopoulos $C$ : Which way does the citric acid cycle turn during hypoxia? The critical role of $\alpha$-ketoglutarate dehydrogenase complex. J Neurosci Res 91 : 1030-1043, 2013

9. Esen F, Erdem T, Aktan D, Kalayci R, Cakar N, Kaya M, et al. : Effects of magnesium administration on brain edema and blood-brain barrier breakdown after experimental traumatic brain injury in rats. J Neurosurg Anesthesiol 15 : 119-125, 2003

10. Fann DY, Lee SY, Manzanero S, Chunduri P, Sobey CG, Arumugam TV : Pathogenesis of acute stroke and the role of inflammasomes. Ageing Res Rev 12 : 941-966, 2013

11. Gallagher CN, Carpenter KL, Grice P, Howe DJ, Mason A, Timofeev I, et al. : The human brain utilizes lactate via the tricarboxylic acid cycle: a 13C-labelled microdialysis and high-resolution nuclear magnetic resonance study. Brain 132(Pt 10) : 2839-2849, 2009

12. Helmy MM, Helmy MW, El-Mas MM : Additive renoprotection by pioglitazone and fenofibrate against inflammatory, oxidative and apoptotic manifestations of cisplatin nephrotoxicity: modulation by PPARs. PLoS One 10 : e0142303, 2015

13. Huang Q, Sun M, Li M, Zhang D, Han F, Wu JC, et al. : Combination of NAD+ and NADPH offers greater neuroprotection in ischemic stroke models by relieving metabolic stress. Mol Neurobiol 55 : 6063-6075, 2018

14. Hsu CP, Oka S, Shao D, Hariharan N, Sadoshima J : Nicotinamide phosphoribosyltransferase regulates cell survival through NAD+ synthesis in cardiac myocytes. Circ Res 105 : 481-491, 2009

15. Irrera N, Pizzino G, Calò M, Pallio G, Mannino F, Famà F, et al. : Lack of the Nlrp3 inflammasome improves mice recovery following traumatic brain injury. Front Pharmacol 8 : 459, 2017

16. Izawa $Y$, Takahashi S, Suzuki N : Pioglitazone enhances pyruvate and lactate oxidation in cultured neurons but not in cultured astroglia. Brain Res 1305 : 64-73, 2009

17. Katsuki $\mathrm{H}$ : Exploring neuroprotective drug therapies for intracerebral hemorrhage. J Pharmacol Sci 114 : 366-378, 2010

18. Keep RF, Hua Y, Xi G : Intracerebral haemorrhage: mechanisms of injury and therapeutic targets. Lancet Neurol $11:$ 720-731, 2012

19. Kim SJ, Kim SH, Kim JH, Hwang S, Yoo HJ : Understanding metabolomics in biomedical research. Endocrinol Metab (Seoul) 31 : 7-16, 2016

20. Lebeaupin C, Proics E, de Bieville CH, Rousseau D, Bonnafous S, Patouraux $S$, et al. : ER stress induces NLRP3 inflammasome activation and hepatocyte death. Cell Death Dis 6 : e1879, 2015

21. Li Y, Yang J, Chen MH, Wang Q, Qin MJ, Zhang T, et al. : Ilexgenin A inhibits endoplasmic reticulum stress and ameliorates endothelial dysfunction via suppression of TXNIP/NLRP3 inflammasome activation in an AMPK dependent manner. Pharmacol Res 99 : 101-115, 2015

22. Lu A, Tang Y, Ran R, Ardizzone TL, Wagner KR, Sharp FR : Brain genom- 
ics of intracerebral hemorrhage. J Cereb Blood Flow Metab 26 : 230-252, 2006

23. Mayer SA, Rincon F : Treatment of intracerebral haemorrhage. Lancet Neurol $4: 662-672,2005$

24. Ribo $M$, Grotta JC : Latest advances in intracerebral hemorrhage. Curr Neurol Neurosci Rep 6 : 17-22, 2006

25. Rice AC, Zsoldos R, Chen T, Wilson MS, Alessandri B, Hamm RJ, et al. : Lactate administration attenuates cognitive deficits following traumatic brain injury. Brain Res 928 : 156-159, 2002

26. Ricote M, Li AC, Willson TM, Kelly CJ, Glass CK : The peroxisome proliferator-activated receptor- $y$ is a negative regulator of macrophage activation. Nature 391 : 79-82, 1998

27. Rolland WB 2nd, Manaenko A, Lekic T, Hasegawa Y, Ostrowski R, Tang J, et al. : FTY720 is neuroprotective and improves functional outcomes after intracerebral hemorrhage in mice. Acta Neurochir Suppl 111 : 213-217, 2011

28. Rynkowski MA, Kim GH, Komotar RJ, Otten ML, Ducruet AF, Zacharia $B E$, et al. : A mouse model of intracerebral hemorrhage using autologous blood infusion. Nat Protoc 3 : 122-128, 2008

29. Sahuquillo J, Merino MA, Sánchez-Guerrero A, Arikan F, Vidal-Jorge M, Martínez-Valverde $T$, et al. : Lactate and the lactate-to-pyruvate molar ratio cannot be used as independent biomarkers for monitoring brain energetic metabolism: a microdialysis study in patients with traumatic brain injuries. PLoS One 9 : e102540, 2014

30. Sánchez-Abarca LI, Tabernero A, Medina JM : Oligodendrocytes use lactate as a source of energy and as a precursor of lipids. Glia 36 : 321329, 2001

31. Schroder K, Zhou R, Tschopp J : The NLRP3 inflammasome: a sensor for metabolic danger? Science 327 : 296-300, 2010

32. Song N, Liu ZS, Xue W, Bai ZF, Wang QY, Dai J, et al. : NLRP3 phosphorylation is an essential priming event for inflammasome activation. Mol Cell 68 : 185-197.e6, 2017

33. Strbian D, Kovanen PT, Karjalainen-Lindsberg ML, Tatlisumak T, Lindsberg PJ : An emerging role of mast cells in cerebral ischemia and hemor- rhage. Ann Med 41 : 438-450, 2009

34. Stutz A, Kolbe CC, Stahl R, Horvath GL, Franklin BS, van Ray O, et al. : NLRP3 inflammasome assembly is regulated by phosphorylation of the pyrin domain. J Exp Med 214 : 1725-1736, 2017

35. Timofeev I, Carpenter KL, Nortje J, Al-Rawi PG, O'Connell MT, Czosnyka $\mathrm{M}$, et al. : Cerebral extracellular chemistry and outcome following traumatic brain injury: a microdialysis study of 223 patients. Brain 134(Pt 2) : 484-494, 2011

36. Vander Heiden MG, Cantley LC, Thompson CB : Understanding the Warburg effect: the metabolic requirements of cell proliferation. Science 324 : 1029-1033, 2009

37. Wang $\mathrm{J}$ : Preclinical and clinical research on inflammation after intracerebral hemorrhage. Prog Neurobiol 92 : 463-477, 2010

38. Wang J, Doré $S:$ Inflammation after intracerebral hemorrhage. J Cereb Blood Flow Metab 27 : 894-908, 2007

39. Wang J, Song MY, Lee JY, Kwon KS, Park BH : The NLRP3 inflammasome is dispensable for ER stress-induced pancreatic $\beta$-cell damage in Akita mice. Biochem Biophys Res Commun 466 : 300-305, 2015

40. Wang Y, Yu B, Wang L, Yang M, Xia Z, Wei W, et al. : Pioglitazone ameliorates glomerular NLRP3 inflammasome activation in apolipoprotein $\mathrm{E}$ knockout mice with diabetes mellitus. PLoS One 12 : e0181248, 2017

41. Yao ST, Cao F, Chen JL, Chen W, Fan RM, Li G, et al. : NLRP3 is required for complement-mediated caspase-1 and IL-1beta activation in ICH. J Mol Neurosci 61 : 385-395, 2017

42. Ying W, Wei G, Wang D, Wang Q, Tang $X$, Shi J, et al. : Intranasal administration with NAD+ profoundly decreases brain injury in a rat model of transient focal ischemia. Front Biosci 12 : 2728-2734, 2007

43. Zhao Y, Li Q, Zhao W, Li J, Sun Y, Liu K, et al. : Astragaloside IV and cycloastragenol are equally effective in inhibition of endoplasmic reticulum stress-associated TXNIP/NLRP3 inflammasome activation in the endothelium. J Ethnopharmacol 169 : 210-218, 2015

44. Zhu X, Tao L, Tejima-Mandeville E, Qiu J, Park J, Garber K, et al. : Plasmalemma permeability and necrotic cell death phenotypes after intracerebral hemorrhage in mice. Stroke 43 : 524-531, 2012 